\title{
Defeito Completo do Septo Atrioventricular com Cianose
}

\author{
Complete Repair in Total Atrioventricular Canal Defect with Cyanosis
}

Carla Tanamati, Renata Lure Suguimoto, Edmar Atik, Solange Gimenez Copolla, Filomena Regina
Galles, Vera Demarchi Aiello, Miguel Lorenzo Barbero-Marcial
Instituto do Coração do Hospital das Clinicas - FMUSP - São Paulo, SP

Os defeitos do septo atrioventricular total (DSAVT) representam $4 \%$ das mal formações cardíacas e acima de $50 \%$ dos defeitos observados na síndrome de Down $(\mathrm{SD})^{1}$. A apresentação clínica é de insuficiência cardíaca precoce na infância e hipertensão pulmonar por hiperfluxo. Raramente a cianose é observada e sugere hipertensão pulmonar ou associação à tetralogia de Fallot ${ }^{3}$, dupla via de saída de ventrículo direito ${ }^{2}$, anomalia de Ebstein ${ }^{4}$, drenagem anômala de cava esquerda persistente em átrio esquerdo (Barbero Marcial, comunicação pessoal). Crianças com SD são particularmente difíceis de avaliação por apresentarem obstrução de vias aéreas superiores, que podem contribuir com o aumento da resistência pulmonar observada no cateterismo cardíaco. A presença de cianose pré-operatória constitui-se um desafio ao tratamento cirúrgico devido ao risco de hipertensão pulmonar irreversível com falência ventricular direita com, a correção dos defeitos intracardíacos.

Cianose moderada (saturação de $75 \%$ ao ar ambiente) em uma paciente de 7 meses, portadora de síndrome de Down e defeito total do septo atrioventricular (DTSAV). Apesar da cianose a paciente ainda apresentava sinais de hiperfluxo pulmonar como dispnéia, hepatomegalia e sopro sistólico em borda esternal esquerda. Cateterismo cardíaco demonstrou $\mathrm{Qp} / \mathrm{Qs}=1,67$ e resistência vascular pulmonar em $6,5 \mathrm{U} / \mathrm{m}^{2}$.

Foi deixado aberto um pequeno forame oval. Após a circulação extracorpórea, a saturação de oxigênio era de $80 \%\left(\mathrm{FiO}_{2}\right.$ 100\%, NO 20 ppm). Ủma biópsia pulmonar foi feita no terceiro dia de pós-operatório quando o tórax foi fechado e revelou lesões de hipertensão pulmonar tipo Heath-Edwards grade III.

O óxido nítrico foi mantido até o sexto dia de pósoperatório e um bloqueador de canal de cálcio foi administrado com melhora da oximetria. Após um longo período de pós-operatório devido à insuficiência cardíaca congestiva e infecção, a paciente recebeu alta sem cianose com classe funcional II (NYHA).

\section{ReLATO do CAso}

Lactente do sexo feminino, de 7 meses com síndrome de Down e história de sopro cardíaco desde o nascimento, taquipnéia e baixo ganho ponderal. Havia sido hospitalizada previamente por broncopneumonia quando foi observada cianose (sat $\mathrm{O}_{2} 80 \%-85 \%$ ) em ar ambiente. 0 ecocardiograma mostrou defeito total do septo

\section{Palavra-chave}

Septo atrioventricular total, cyanosis, correção total.
Atrioventricular septal defects account for $4 \%$ of congenital cardiac malformations and over $50 \%$ of cardiac defects seen in Down syndrome ${ }^{1}$. Clinical presentation is marked by congestive heart failure early in infancy. Cyanosis is rarely found in infants and suggests irreversible pulmonary hypertension or associated cardiac defects as tetralogy of Fallot, double outlet right ventricle ${ }^{2}$, Ebstein anomaly ${ }^{3}$, persistent left superior vena cava draining in the left atrium (Barbero Marcial, personal communication). Children with Down's syndrome is particularly difficult to assess because they often suffer from upper airways obstruction ${ }^{4}$, which may contribute to the increased pulmonary vascular resistance determined at cardiac catheterization. This association of factors becomes a challenge for operability and, we will report one such case.

Correspondência: Carla Tanamati - Rua Correia de Lemos, 244/143 - 04140-000 - São Paulo, SP E-mail: ctanama@terra.com.br

atrioventricular com grande comunicação interventricular (CIV), valva atrioventricular comum com regurgitação moderada e uma comunicação interatrial tipo ostium primum (OP). A pressão pulmonar sistólica foi estimada pelo eco em $53 \mathrm{mmHg}$.

No exame clínico havia cianose moderada (sat $0,70 \%$ $80 \%$ ), um sopro sistólico alto na borda esternal esquerda e hepatomegalia.

O eletrocardiograma mostrava ritmo sinusal, com sinais de sobrecarga de ventrículo direito. Na radiografia de tórax havia cardiomegalia moderada e aumento da vascularização pulmonar. A angiografia pulmonar era normal (fig. 1), a resistência vascular pulmonar era de

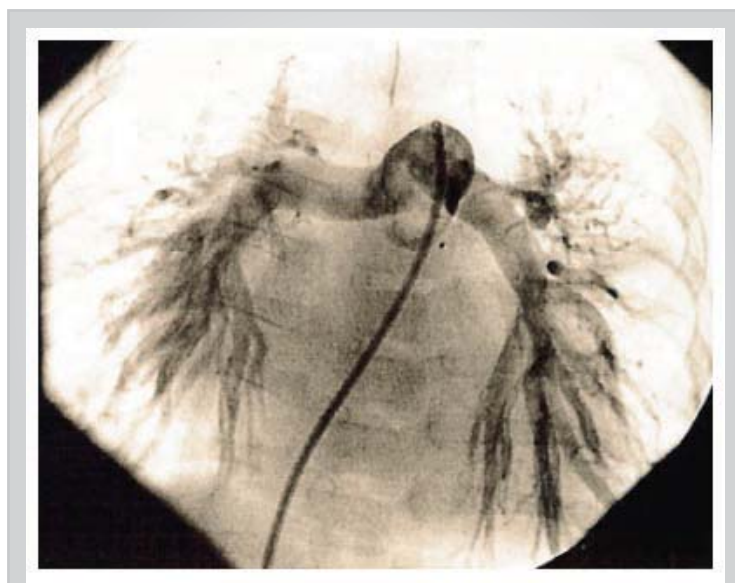

Fig. 1 - Angiograma pulmonar pré-operatório. Recebido em 22/03/05 - Aceito em 22/08/05 


\section{6,5 U/m², com Qp/Qs 1,67( $\left.\mathrm{FiO}_{2}=100 \%\right)$.}

A correção total foi feita com circulação extracorpórea (CEC) hipotérmica a $20^{\circ} \mathrm{C}$. A CIV foi fechada com pericárdio bovino. A valva átrio ventricular foi dividida sem regurgitação e o OP foi fechado com pericárdio autólogo. Um forame oval de $4 \mathrm{~mm}$ foi deixado aberto, devido a elevada pressão pulmonar. Após a CEC o Eco transesofágico mostrou uma valva AV competente, ausência de CIA ou CIV residuais e um pequeno shunt direito-esquerdo pelo forame oval. Em ritmo sinusal a saturação de $\mathrm{O}_{2}$ era $80 \%$ (FiO2 100\%; NO 20ppm). $\mathrm{O}$ esterno foi deixado aberto e um cateter de artéria pulmonar (AP) foi colocado através do ventrículo direito (VD).

O esterno foi fechado no terceiro dia de pós-operatório e uma biópsia pulmonar foi feita devido a elevada pressão de AP (nível sistêmico), com crises de HP e hipoxemia revelando um grau III da classificação de Heath-Edwards (fig. 2).

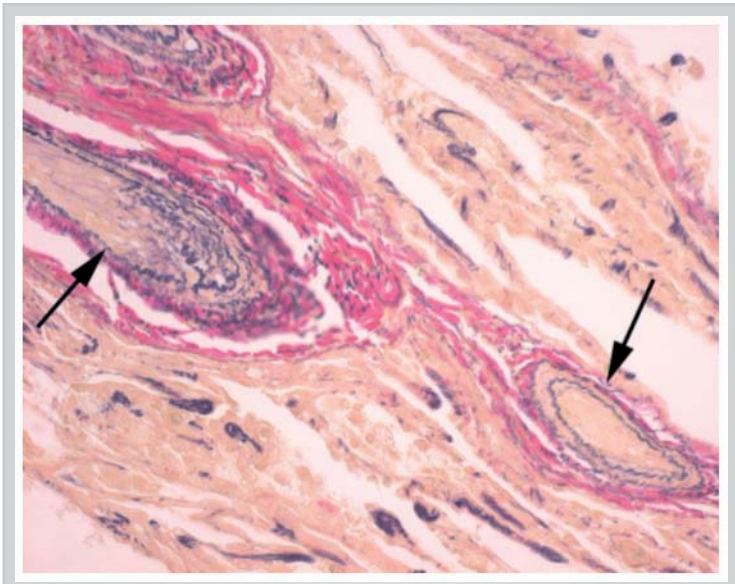

Fig. 2 - Biópsia pulmonar - Microfotografia da biópsia pulmonar mostrando 2 arteríolas intra-acinares com obstrução completa por proliferação celular (setas escuras). Coloração de Miller para fibras elásticas. Aumento de 40x.

No sexto dia de pós-operatório foi introduzido um bloqueador de canal de cálcio (nifedipina) com melhora da sat de $\mathrm{O}_{2}, 96 \%$.

Apresentou broncopneumonia, tratada com cefepime e vancomicina, além de uremia, e ascite, responsivas a diuréticos.

Após duas tentativas, a criança foi extubada no vigésimo dia de pós-operatório e as drogas vasoativas suspensas. Evoluiu com picos febris e com hemoculturas e cultura de ponta de cateter positivas para Candida albicans, sendo iniciado anfotericina B por 21 dias.

Recebeu alta no $48^{\circ}$ dia de internação sem dispnéia ou cianose, em uso de nifedipina, furosemida e L tiroxina.

\section{Discussão}

A avaliação pré-operatória da hipertensão pulmonar e a operabilidade destes casos é frequëntemente difícil ${ }^{5}$. A história clínica fornece dados importantes, pois o aumento da resistência vascular pulmonar leva à melhora da insuficiência cardíaca (menor shunt pela CIA e CIV). A radiografia de tórax mostra sinais de hiperfluxo pulmonar na resistência pulmonar baixa e quando há doença obstrutiva apresenta hipertranslucência e dilatação dos vasos hilares. 0 cateterismo cardíaco é utilizado para medida da resistência vascular pulmonar e angiografia.

A cianose ou oximetria $\leq 92 \%$ foi observada em $31 \%$ de 77 neonatos com síndrome de Down ${ }^{6}$, e foi associada com outras cardiopatias como tetralogia de Fallot (11\%) e tetralogia de Fallot com DSAV (2\%). A presença de cianose moderada (sat $\mathrm{O}_{2} 80 \%-85 \%$ ) em pacientes com DSAV é sugestiva de doença vascular pulmonar obstrutiva e a operabilidade deve ser extensamente estudada no préoperatório. 0 cateterismo cardíaco com angiografia, Qp/Qs e cálculo da resistência vascular pulmonar com e sem vasodilatadores $\left(\mathrm{O}_{2}, \mathrm{NO}\right.$, prostaglandina) é obrigatório. Se o paciente ainda tem sintomas de insuficiência cardíaca (taquipnéia, pouco ganho ponderal, hepatomegalia) e o cateterismo cardíaco mostra angiografia normal, Qp/Qs $=1,67$, e RVP $=6,5 \mathrm{U} / \mathrm{m}^{2}$, o tratamento cirúrgico deve ser feito, apesar da cianose e resistência elevada.

A correção total foi feita neste caso deixando um forame oval aberto para descomprimir o coração direito. Óxido nítrico (10ppm), 100\% $\mathrm{FiO}_{2}$ e milrinone foram administrados ao final da CEC e substituídos por bloqueadores de canal de cálcio para controlar as crises de hipertensão pulmonar (tab. 1). A biópsia pulmonar deve

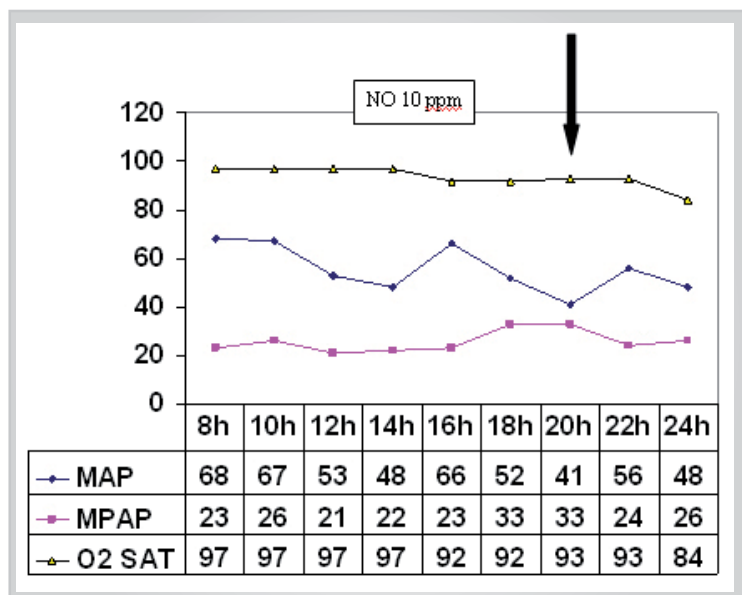

MAP- pressão arterial média ( $\mathrm{mmHg}$ ); MPAP- pressão arterial pulmonar média $(\mathrm{mmHg})$; 02 sat - saturação de oxigênio.

Gráfico 1 - Representação de uma crise de hipertensão pulmonar no $5^{\circ}$ dia do pós-operatório.

ser feita para indicar operabilidade na suspeita de doença vascular pulmonar-9 apesar de ter sido feita no terceiro pós-operatório quando foi fechado o esterno. A regressão da hipertensão pode ser relacionada ao crescimento do leito vascular ${ }^{10}$, porém, é obrigatório a monitorização da pressão pulmonar.

Os pacientes com defeito total do septo atrioventricular com índice de resistência vascular pulmonar menor que 6 $\mathrm{U} / \mathrm{m}^{2}$ podem ser considerados candidatos ao tratamento cirúrgico se ainda apresentam sinais de shunt esquerda- 
direita. O seguimento a longo prazo de pacientes com CIV e resistência superior a $6 \mathrm{U} / \mathrm{m}^{2}$ tem mostrado bons resultados ${ }^{11}$ em 31 (79\%) com redução significante da pressão pulmonar. Uma análise ampla dos dados clínicos e laboratoriais prévios a cirurgia pode guiar o manejo adequado.

\section{Potencial Conflito de Interesses}

Declaro não haver conflitos de interesses pertinentes.

\section{REFERÊNCIAS}

1. Mitchell SC, Korones SB, Berendes HW. Congenital heart disease in 56,109 live births. Incidence and natural history. Circulation. 1971; 43: 323.

2. Clapp S, Perry BL, Farooki ZQ, et al. Down's syndrome, complete atrioventricular canal, and pulmonary vascular obstructive disease. J Thorac Cardiovasc. 1990; 110: 115-121.

3. Bharati S, Kirklin JW, McAllister HA Jr, et al. The surgical anatomy of common atrioventricular orifice associated with tetralogy of Fallot, double outlet right ventricle and complete regular transposition. Circulation. 1980; 61: 1142-9.

4. Guenthard J, Wyler F. Complete atrioventricular septal defect and Ebstein anomaly. Pediatr Cardiol. 1996; 17: 67-9.

5. Haworth SG. Pulmonary vascular bed in children with complete atrioventricular septal defect: relation between structural and hemodynamic abnormalities. Am J Cardiol. 1986; 57: 833-9.

6. McElhinney DB, Straka M, Goldmuntz E, Zackai EH. Correlation between abnormal cardiac physical examination and echocardiographic findings in neonates with Down syndrome. Am J Med Genetics. 2002; 113: 238-41.

7. Yamaki S, Yasui $\mathrm{H}$, Kado H, Yonenaga $\mathrm{K}$, et al. Pulmonary vascular disease and operative indications in common atrioventricular canal defect in early infancy. J Thorac Cardiovasc Surg. 1993; 106: 398405.

8. Friedman WF, Heiferman MF. Clinical problems of postoperative pulmonary vascular disease. Am J Cardiol. 1982; 50: 631-6.

9. Guimarães JI, Lopes AA, Martins RF, et al. Guideline for diagnosis, evaluation and therapeutic of pulmonar hypertension. Arq Bras Cardiol. 2003; 81(Suppl 8): 1-10.

10. Egito ES, Aiello V, Bosisio IB, et al. Vascular remodeling processin reversibility of pulmonary arterial hypertension secondary to congenital heart disease. Pathol Res Pract. 2003; 199: 521-32.

11. Kannan BR, Sivasankaran S, Tharakan JA, et al. Long-term outcome of patients operated for large ventricular septal defects with increased pulmonary vascular resistance. Indian Heart J. 2003; 55: 161-6. 